\title{
Enhanced transport through desorption-mediated diffusion
}

\author{
Félix Rojo, ${ }^{1}$ Carlos E. Budde, Jr., ${ }^{1}$ Horacio S. Wio, ${ }^{2}$ and Carlos E. Budde ${ }^{1}$ \\ ${ }^{1}$ Fa.M.A.F., Universidad Nacional de Córdoba, Ciudad Universitaria, X5000HUA Córdoba, Argentina \\ ${ }^{2}$ Instituto de Física de Cantabria, Universidad de Cantabria and CSIC, E-39005 Santander, Spain
}

(Received 31 October 2012; published 16 January 2013)

\begin{abstract}
We present a master equation approach to the study of the bulk-mediated surface diffusion mechanism in a threedimensional confined domain. The proposed scheme allowed us to evaluate analytically a number of magnitudes that were used to characterize the efficiency of the bulk-mediated surface transport mechanism, for instance, the mean escape time from the domain, and the mean number of distinct visited sites on the confined domain boundary.
\end{abstract}

DOI: 10.1103/PhysRevE.87.012115

PACS number(s): $05.40 . \mathrm{Fb}$

\section{INTRODUCTION}

Intermittent processes are widespread in many domains and fields. Generally speaking, these processes involve an agent (particle, searcher, etc.) and two or more phases among which it alternates. The importance of these processes is based on its capacity to explain several physical scenarios, ranging from animal search patterns [1], through the solutions or melts of synthetic macromolecules [2], to the manufacture of selfassembled mono- and multilayers [3]. This sort of intermittent behavior is also found in the binding of a protein to specific sites on DNA for regulating transcription [4], or in interface sciences (see [5] and references therein) where the adsorptiondesorption dynamics of molecules are of fundamental importance and are crucial to a number of technologies. Among these the adsorption-desorption mechanism called bulk-mediated diffusion [5] arises at interfaces separating a liquid bulk phase and a second phase which may be either solid, liquid, or gaseous. Whenever the adsorbed species is soluble in the liquid bulk, adsorption-desorption processes occur continuously. These processes generate a surface displacement because molecules desorb, undergo Fickian diffusion in the bulk liquid, and are then re-adsorbed elsewhere. As this process repeats over time, it results in an effective diffusion of a molecule on the surface. A number of magnitudes have become available, due to the improvements on measurement techniques [6], and might serve to characterize the efficiency of the bulk-mediated transport mechanism. For instance, a technique named TIRFM (total internal reflectance fluorescence microscopy) [6] allows an observer to track down the trajectories of single molecules and thus the span, due to the mixed transport, over the surface. Furthermore such technique could provide the time spent on each surface-bulk phase, and hence the overall time until some triggering effect takes place; such an effect may be, for example, the exocytosis in neurons [7]. This last situation, i.e., the escape of a particle from a domain, is usually referred to as the narrow escape time problem (NET) [8].

In [9], we have introduced an analytical Markovian twodimensional (2D) model that showed the impact of geometrical parameters and the interplay between surface and boundary paths in the studied confining domain, for both the perfect and imperfect trapping case [10]. In those works we presented phase diagrams which showed that some combinations of the geometrical parameter and the transport mechanism were required for the existence of an optimal transport.
It is challenging to move forward into the study of the three-dimensional (3D) bounded geometry instance. Such studies would be relevant to heterogeneous catalysis and molecular recognition associated with both biomembranes and biosensors [6,11], just to mention a few examples. Recently the continuous version of this problem has been tackled [12]. In this work we introduce a 3D bounded lattice geometry which allows a complete exact solution. We also introduce an imperfect trap site (or narrow escape window [13]) on the surface, which enabled us to measure the efficiency of the mixed transport mechanism under imperfect trapping conditions. It is well known that systems' descriptions by means of the "imperfect trapping case" are suitable whenever the surface contains "deep traps," or when there are captures and re-emissions from a surface that contains sites with several internal states (such as the "ladder trapping model"), etc. [14-16]. We believe that the conclusions drawn in this work are qualitatively robust and will contribute to a better understanding of the role played by geometrical and diffusive parameters in the transport through desorption-mediated diffusion.

\section{ANALYTICAL APPROACH}

Let us consider the problem of a walker making a nearest neighbors random walk in a finite $N \times M \times(L+1)$ lattice (see Fig. 1). The bulk is bounded in the $z$ direction where the walkers can move from $z=0$ to $z=L$, and periodic boundary conditions are assumed in the $x$ and $y$ directions, so, for instance, $x$ and $x+N$ denote the same place in space. A complete and finite set of equations can be constructed for the walker's unrestricted conditional probability $P\left(n, m, l, t \mid n_{0}, m_{0}, l_{0}, t=0\right)$ of being in position $(n, m, l)$ at time $t$ given that it departed from $\left(n_{0}, m_{0}, l_{0}\right)$ at $t=0$. By resorting to the matrix formalism ( [5]) this set can be written in the Fourier[ $\left[(x, y) \rightarrow\left(k_{x}, k_{y}\right)\right]$-Laplace $(t \rightarrow u)$ space [17] as $[u \mathbb{I}-\mathbb{H}] \mathbb{P}=\mathbb{I}$, where $\mathbb{I}$ is the identity matrix, $\mathbb{H}$ is a $(L+1) \times(L+1)$ tridiagonal matrix with elements:

$$
\mathbb{H}=\left[\begin{array}{cccccc}
C_{1} & \gamma & 0 & \ldots & \cdots & 0 \\
\delta & C & \gamma & 0 & \cdots & 0 \\
0 & \gamma & C & \gamma & 0 & \vdots \\
\cdots & 0 & \ddots & \ddots & \ddots & \vdots \\
\cdots & \cdots & \cdots & \gamma & C & \gamma \\
0 & \cdots & & 0 & \gamma & \gamma+C
\end{array}\right],
$$




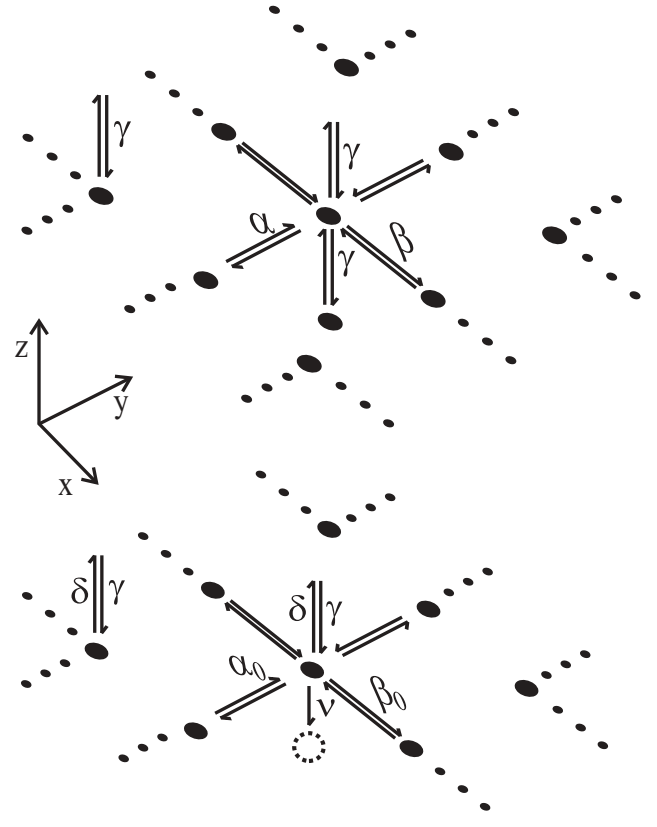

FIG. 1. Schematic system. $\gamma$ is the surface transition probability per unit time in the $z$ direction, $\alpha\left(\alpha_{0}\right)$ and $\beta\left(\beta_{0}\right)$ are the bulk $(z=0$ surface) transition probabilities in the $x$ and $y$ direction respectively, and $\delta$ is the desorption probability per unit time from the boundary surface $z=0$. The entrance to the trap or escape site (empty circle) is regulated by the transition rate $v$.

where constants $C$ and $C_{1}$ are defined as $C=-2 \gamma+$ $A_{k}=-2 \gamma+2 \alpha\left(\cos k_{x}-1\right)+2 \beta\left(\cos k_{y}-1\right), \quad C_{1}=-\delta+$ $A_{k}^{0}=-\delta+2 \alpha_{0}\left(\cos k_{x}-1\right)+2 \beta_{0}\left(\cos k_{y}-1\right)$, and $\mathbb{P}$ is a $(L+1) \times(L+1)$ matrix with components $\left[\mathbb{P}\left(k_{x}, k_{y}, u\right)\right]_{l, l_{0}}=$ $P\left(k_{x}, k_{y}, l, u \mid n_{0}, m_{0}, l_{0}, t=0\right)$. By applying the Dyson procedure ([5]) a general expression for $\left[\mathbb{P}\left(k_{x}, k_{y}, u\right)\right]_{l, l_{0}}$ can be found. It is worth mentioning that desorption from the surface at $z=0$ is regulated by the transition rate $\delta$, so this parameter enables us to regulate the "exchange rate" between surface and bulk paths. We also incorporate an imperfect trapping site at the surface, where the walker may escape from the domain. For this, we define $A\left(\vec{s}, t \mid \vec{s}_{0}, 0\right)$ as the absorption (trapping) probability density (APD) through the site $\vec{s}$ at time $t$, given that the walker was at $\vec{s}_{0}$ at time $t=0$. Hence $A\left(\vec{s}, t \mid \vec{s}_{0}, 0\right) d t$ gives the trapping probability of the walker, through $\vec{s}$ and between $t$ and $t+d t$, given that it departed at $t=0$ from $\vec{s}_{0}$. The connection between the APD and the unrestricted conditional probability $P\left(\vec{s}, t \mid \vec{s}_{0}, t=0\right)$ can be traced to results in [15]; this approach in the Laplace domain gives

$$
A\left(\vec{s}, u \mid \vec{s}_{0}, t=0\right)=\frac{v P\left(\vec{s}, u \mid \vec{s}_{0}, t=0\right)}{1+v P(\overrightarrow{0}, u \mid \overrightarrow{0}, t=0)},
$$

where $v$ introduces the imperfection in the trapping process. As this parameter ranges from 0 to $\infty$, it allows the study of both a deficient trapping and situations where escape is certain (i.e., perfect trapping case).

Once $A(\cdot)$ is obtained, a number of magnitudes that may be helpful to characterize the mixed transport can be evaluated, for example, the moments of the APD can be obtained as

$$
\begin{aligned}
\left\langle t^{i}(\vec{s})\right\rangle & =\int_{0}^{\infty} t^{i} \sum_{\vec{s}_{0}} A\left(\vec{s}, t \mid \vec{s}_{0}, 0\right) g\left(\vec{s}_{0}\right) d t \\
& =\left.(-1)^{i} \frac{\partial^{i}}{\partial u^{i}}\left\{\sum_{\vec{s}_{0}} A\left(\vec{s}, u \mid \vec{s}_{0}, 0\right) g\left(\vec{s}_{0}\right)\right\}\right|_{u=0},
\end{aligned}
$$

where $g\left(\vec{s}_{0}\right)$ denotes the probability density of initially finding the walker at position $\vec{s}_{0}$ [18]. For instance, the mean absorption/escape time, i.e., the mean time until the walker is absorbed through site $\vec{s}$, is $\langle t(\vec{s})\rangle$. Furthermore, another measurable quantity is the mean number of distinct visited sites $S$, a magnitude that has been proved to be reliable and useful in other scenarios such as intermittent searching [1], and could be used as a measure of the efficiency with which the walker scans the surface. It is worth noting that $S$ can be used to characterize the process only for the perfect trapping case, i.e., it does not take into account the trapping process itself. In the Laplace space the mean number of distinct visited sites evaluates to

$$
S(u)=\frac{1}{u} \sum_{\vec{s}} \frac{P\left(\vec{s}, u \mid \vec{s}_{0}, t=0\right)}{P(\overrightarrow{0}, u \mid \overrightarrow{0}, t=0)} .
$$

This quantity could be used, for example, to complement or contrast the information obtained from $\langle t(\vec{s})\rangle$.

\section{RESULTS AND ILLUSTRATIONS}

\section{A. Results}

Here we present the results and illustrate the general framework introduced in the previous paragraphs. The results shown in this work may be classified as analytical (in coordinate space and in the Laplace domain), analyticalnumerical (results in coordinate space and time domain-after performing the Laplace inversion numerically) and Monte Carlo (MC) simulations [19]. In the following we evaluate the mean escape time through site $\vec{s}=(0,0,0)$ [we adopt the notation $\langle t(\overrightarrow{0})\rangle \rightarrow\langle t\rangle]$ for a walker that starts at site $\left(0,0, l_{0}\right)$ [20], i.e., $g(n, m, l)=\delta_{n, 0} \delta_{m, 0} \delta_{l, l_{0}}$, and obtain

$$
\begin{aligned}
\langle t\rangle= & {\left[\frac{(2 L+1)-l_{0}}{2 \gamma}\right] l_{0}+\left[\frac{\delta L}{\gamma}+1\right] } \\
& \times\left\{\frac{N M}{v}+\sum_{\left(k_{x}, k_{y}\right)^{\prime}}\left[\mathbb{P}\left(k_{x}, k_{y}, 0\right)\right]_{0,0}-\left[\mathbb{P}\left(k_{x}, k_{y}, 0\right)\right]_{0, l_{0}}\right\},
\end{aligned}
$$

where

$$
\left[\mathbb{P}\left(k_{x}, k_{y}, u\right)\right]_{0, l_{0}}=\frac{\eta^{l_{0}}+\eta^{\tilde{L}-l_{0}}}{\delta(1-\eta)\left(1-\eta^{\tilde{L}-1}\right)+\left(u-A_{k}^{0}\right)\left(1+\eta^{\tilde{L}}\right)},
$$

$k_{x}=2 \pi q_{1} / N, k_{y}=2 \pi q_{2} / M, q_{1}=0 \ldots N-1, q_{2}=0 \ldots$ $M-1$ [the $\left(k_{x}, k_{y}\right)^{\prime}$ index implies that the $(0,0)$ term is ruled out from the sum], $\tilde{u}=u-A_{k}, \tilde{L}=2 L+1$, and $\eta=$ $1+\left(\tilde{u}-\sqrt{\tilde{u}^{2}+4 \gamma \tilde{u}}\right) / 2 \gamma$. We also present here an analytical calculation of the relative variance or relative error $(\epsilon)$ of $\langle t\rangle$, used to characterize the reliability with which the MET describes the trapping process. We evaluate the relative 
variance as $\epsilon_{\langle t\rangle}=\sqrt{\left\langle t^{2}\right\rangle-\langle t\rangle^{2}} /\langle t\rangle$, where

$$
\begin{aligned}
\left\langle t^{2}\right\rangle= & \left(\frac{N M}{D_{10} v}\right)^{2}+\frac{N M}{D_{10}^{2} v}\left[\left(D_{20}+f_{10}\right)+\Delta D_{2}+\Delta f_{2}\right] \\
& +\frac{1}{D_{10}}\left[\frac{\left(D_{20}+f_{10}\right)\left(\Delta D_{2}+\Delta f_{1}\right)}{D_{10}}-\left(\Delta D_{3}+\Delta f_{2}\right)\right],
\end{aligned}
$$

and all the coefficients are obtained from

$$
\begin{gathered}
{[\mathbb{P}(0,0, u)]_{0, l_{0}}=\frac{D_{1 l_{0}}}{u}+D_{2 l_{0}}+D_{3 l_{0}} u+\cdots} \\
\sum_{\left(k_{x}, k_{y}\right)^{\prime}}\left[\mathbb{P}\left(k_{x}, k_{y}, u\right)\right]_{0, l_{0}}=f_{1 l_{0}}+f_{2 l_{0}} u+f_{3 l_{0}} u^{2}+\cdots,
\end{gathered}
$$

where $D_{i j}$ is the $i$ factor of the Laurent series in $u$ of $[\mathbb{P}(0,0, u)]_{0, j}$, and $f_{i j}$ coefficients satisfy $f_{i j}=$ $\left.\frac{\partial^{i-1}}{\partial u^{i-1}}\left\{\sum_{\left(k_{x}, k_{y}\right)^{\prime}}\left[\mathbb{P}\left(k_{x}, k_{y}, u\right)\right]_{0, j}\right\}\right|_{u=0}$. We also define $\Delta D_{i} \equiv$ $D_{i 0}-D_{i l_{0}}$ and $\Delta f_{i} \equiv f_{i 0}-f_{i l_{0}}$. With this notation Eq. (6), e.g., would read

$$
\langle t\rangle=D_{10}^{-1} \Delta D_{2}+D_{10}^{-1}\left\{N M v^{-1}+\Delta f_{1}\right\} .
$$

\section{B. Illustrations}

In the next figures lines indicate analytical calculations, while symbols correspond to Monte Carlo (MC) simulations. We will consider $N=M=20, \alpha=\beta=\gamma=1, l_{0}=L, \alpha_{0}=$ $\beta_{0}=0.1$, and for representation purposes we will draw the scaled MET, $T^{*}=\left(\langle t\rangle-D_{10}^{-1} \Delta D_{2}\right) / N M$.

In Fig. 2 we present the curves corresponding to the scaled MET (mean escape time) as a function of the desorption rate $\delta$, for different values of $L$ (system size in the $z$ direction) for the "perfect trapping case" $(v=\infty)$. As can be inferred from the figure, certain combinations of the system parameters benefit either bulk paths $(L=1)$, a mixed kind of transport-bulk and surface paths $(L=3,5)$, or surface paths $(L=10,15,19)$. By considering the regimes (i) $\delta \ll \alpha_{0}$, (ii) $\delta \approx \alpha_{0}$, and

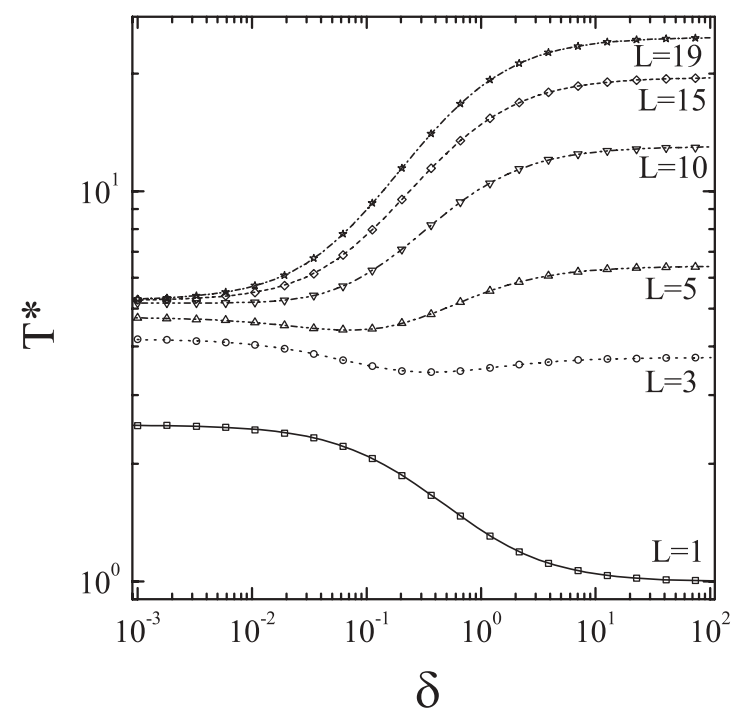

FIG. 2. Scaled MET for the perfect trapping case $(v=\infty)$ as a function of the desorption rate $\delta$ (in $\log$ scale), with $M=N=20$, $\alpha_{0}=0.1$, for different sizes of $L=1,3,5,10,15,19$.

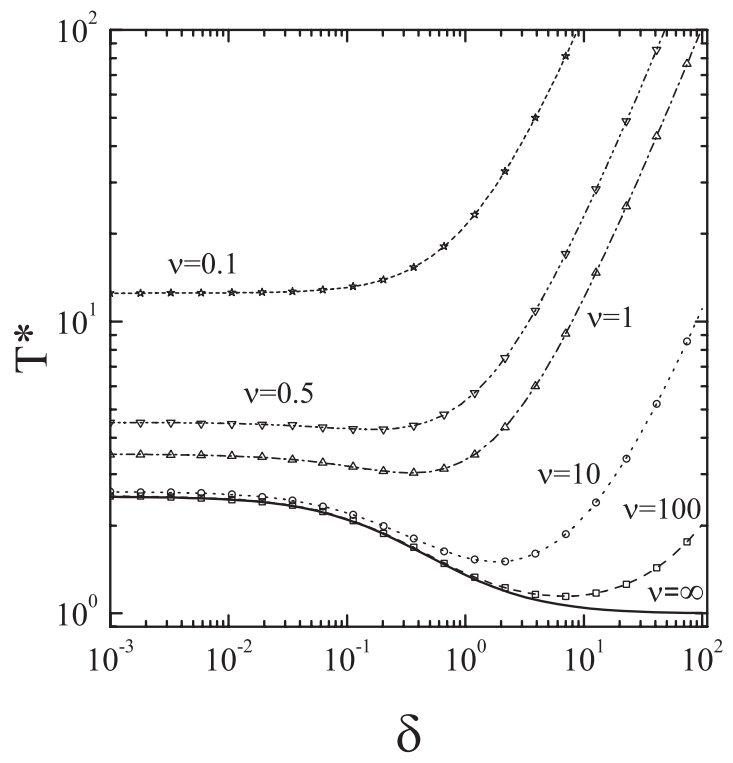

FIG. 3. Scaled MET for the imperfect trapping case as a function of the desorption rate (in $\log$ scale) $\delta$, with $M=N=20, L=1, \alpha_{0}=$ 0.1 , for different values of the imperfection $v=0.1,0.5,1,10,100, \infty$.

(iii) $\delta \gg \alpha_{0}$ in Eq. (5) approximate bounds regarding the existence of a local minimum in MET can be derived,

$$
\left(\frac{1}{L}+\frac{\delta_{\mathrm{i}}}{\gamma}\right) \frac{\left.\Delta f_{1}\right|_{\mathrm{i}}}{\left.\Delta f_{1}\right|_{\mathrm{ii}}}-\frac{1}{L}>\frac{\alpha_{0}}{\gamma}>\frac{\left.\Delta f_{1}\right|_{\mathrm{ii}}}{\left(\left.\Delta f_{1}\right|_{\mathrm{iii}}-\left.\Delta f_{1}\right|_{\mathrm{ii}}\right) L},
$$

where $\left.\Delta f_{1}\right|_{\mathrm{j}}$ is $\Delta f_{1}$ evaluated in regime $j=\mathrm{i}, \mathrm{ii}$,iii [21], and we maintain $\delta \mathrm{i} / \gamma$ since as $L$ grows it may be not neglected. Notice that the bounds in Eq. (11) imply a restriction on the transition rates at the boundary and in the bulk. This result can be put in direct correspondence to those found in the continuous case, Ref. [8] [Eq. (87)], by making the assignments $\alpha_{0} \rightarrow D_{1}$ (diffusion coefficient at the boundary) and $\gamma \rightarrow D_{2}$ (diffusion coefficient in the bulk). Taking into account relation (11) for the parameters considered in the former figure, we observe that the MET should have a local minimum for $3 \leqslant L \leqslant 9$, thus in good agreement with curves depicted in Fig. 2.

In Fig. 3 we present the curves corresponding to the scaled MET, as a function of the desorption rate $\delta$, for $L=1$ and for different values of the "escape strength" $v$, which is the transition rate at the escape window. We have included for comparison the perfect trapping case (continuous thick line). As can be inferred from the figure, $v$ values significantly influence the existence of a minimum in the MET. Changes in the location of the extrema values of the MET range from a monotonous behavior $(\nu \rightarrow \infty$ extrema in $\delta \rightarrow \infty)$ to a situation with a global minimum, and then back again into a monotonous behavior $(v \rightarrow 0$ extrema in $\delta \rightarrow 0)$.

In Fig. 4 we include the relative error $\left(\epsilon_{\langle t\rangle}\right)$ of the MET as a function of $\delta$ for the perfect (a) and imperfect (b) trapping cases. Due to the geometry and transport properties of the proposed system (3D lattice and regular diffusion) this case belong to the noncompact exploration case defined in [22]. In this situation it would be expected that $\epsilon_{\langle t\rangle} \sim 1$, thus consistent with our results $\left(1<\epsilon_{\langle t\rangle}<2\right)$. This suggests that 

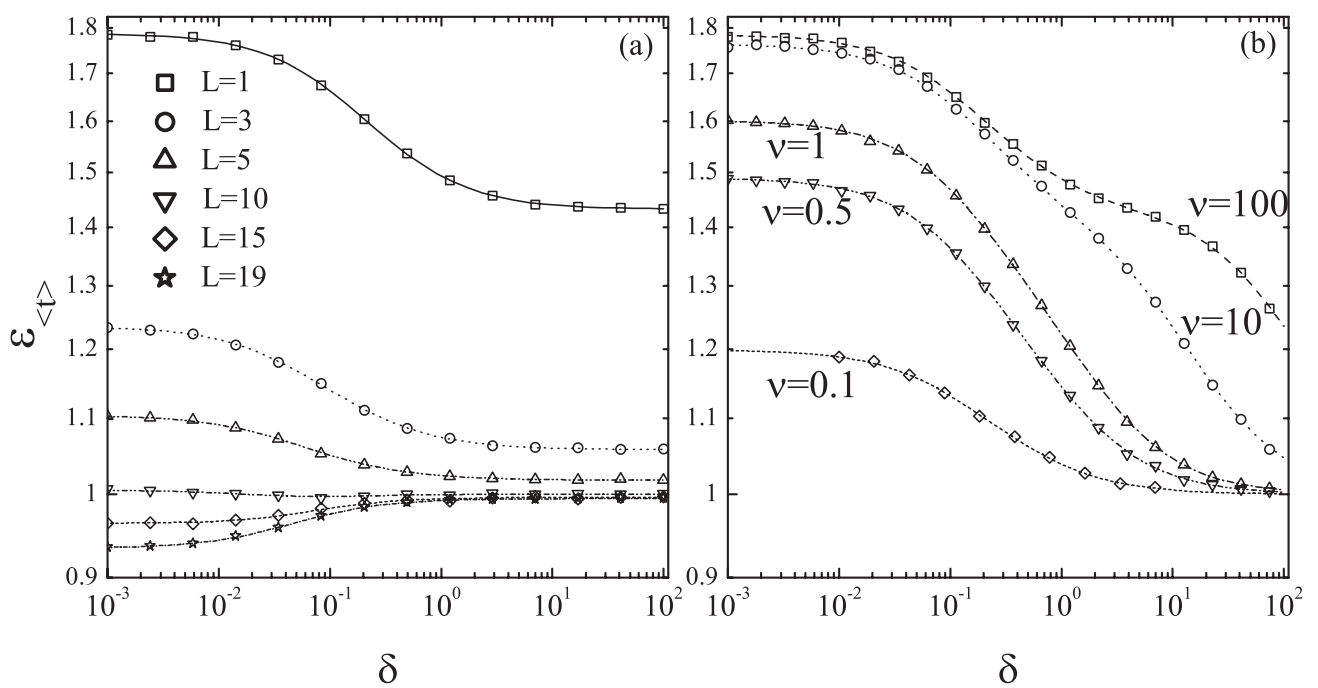

FIG. 4. Relative error $\epsilon_{\langle t\rangle}$ for the perfect (a) and imperfect (b) trapping cases as a function of the desorption rate $\delta$ (in log scale). Parameters of (a) and (b) are the same ones than in Figs. 2 and 3 respectively.

the fluctuations are comparable to what would be obtained with a single parameter distribution [8,22]. In the following figure we introduce a complementary magnitude, the mean number of distinct visited sites on the base surface, $S_{z=0}$.

The mean number of distinct visited sites starting from $\left(0,0, l_{0}\right), S_{z=0}$, evaluates in the Laplace space to

$$
S_{z=0}(u)=\frac{N M}{u} \frac{[\mathbb{P}(0,0, u)]_{0, l_{0}}}{\sum_{\left(k_{x}, k_{y}\right)}\left[\mathbb{P}\left(k_{x}, k_{y}, u\right)\right]_{0,0}} .
$$

In Fig. 5, we plot the scaled $S_{z=0} / N M=S^{*}$ as a function of $\delta$, for different values of $L$, for a fixed time $t_{L}^{\prime}$ [23] (a different one for each curve depending on $L$ ) by performing the Laplace inversion numerically. Notice that $S^{*}$ depicts a similar behavior to the MET (for the perfect trapping case, Fig. 2), and whenever $T^{*}$ has an optimal desorption probability

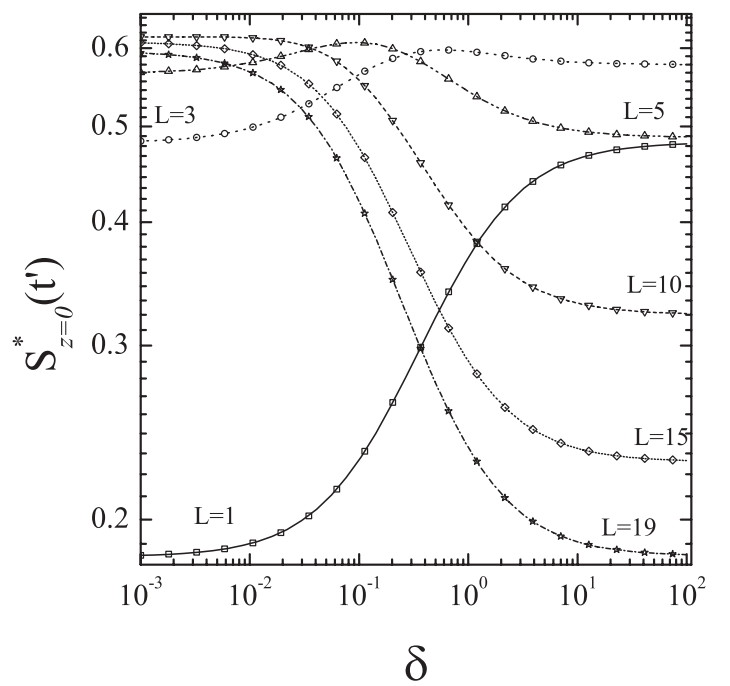

FIG. 5. Scaled mean number of distinct visited sites on the base surface- $S /(N M)$ - for the perfect trapping case as a function of the desorption rate $\delta$ (in $\log$ scale), with $M=N=20, \alpha_{0}=0.1$, for different values of $L$. then $S^{*}$ reflects it. The behavior in the concavity or convexity of the extrema, for example in a maximum, could be interpreted in the following way: in order to find the escape window the walker must "scan" the surface; a maximum in $S_{z=0}$ implies an increase in the number of visited sites (on the surface), increasing the chances of hitting the escape window and thus reducing (a minimum on) the mean escape time $T$.

In Fig. 6 we plot the relative error $\epsilon_{\langle S\rangle}$ of the mean number of distinct visited sites as a function of $\delta$, corresponding to the results of Fig. 5, and obtained via MC simulations. We observe that $\epsilon_{\langle S\rangle}<1$, which are consistent with those results obtained for the continuous system pointed out in [11] (crossing case). Thus with the inclusion of $\epsilon_{\langle S\rangle}$ we complete the presentation of $S$ as a complementary magnitude to characterize the transport through desorption-mediated diffusion, together with $\langle t\rangle$ and its error, $\epsilon_{\langle t\rangle}$.

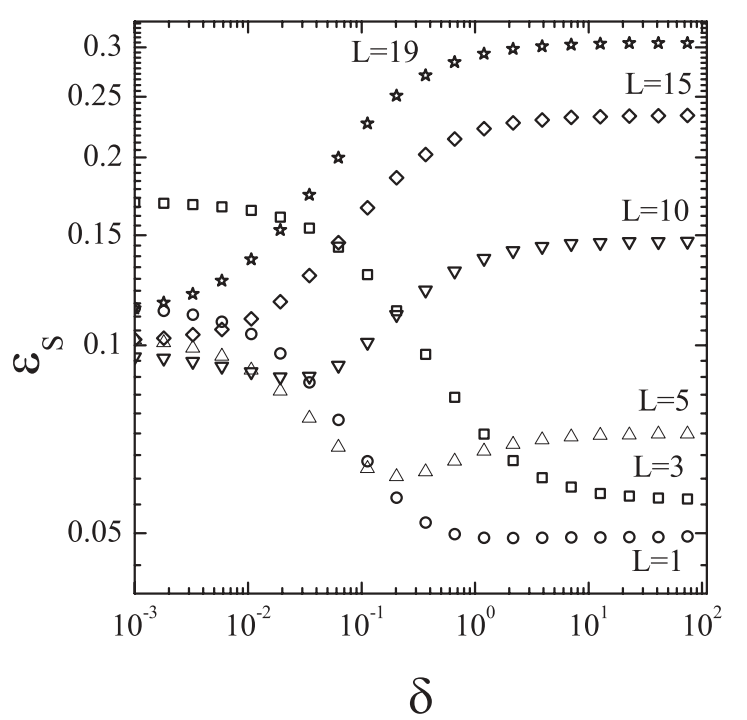

FIG. 6. Relative error of $S, \epsilon_{S}$ as a function of the desorption rate $\delta$ (in $\log$ scale), for $M=N=20, \alpha_{0}=0.1$. All data correspond to MC simulations. 


\section{CONCLUSIONS}

In this work we presented a master equation approach to the study of the bulk-mediated surface diffusion mechanism in a $3 \mathrm{D}$ confined domain. The proposed scheme enabled us to analytically evaluate a number of magnitudes that could be used to characterize the efficiency of the bulk-mediated surface transport mechanism. In addition to the study of the enhanced transport through desorption-mediated diffusion for the perfect trapping case, we have introduced a finite transition probability, namely $v$, at the narrow escape window, that also allowed us to analyze the imperfect escape case.

By resorting to Dyson' technique we have obtained analytic results for the mean escape time (MET) $\langle t\rangle$, for the mean number of distinct sites visited by the walker $S(t)$, for the relative error $\epsilon_{\langle t\rangle}$ and we have performed MC simulation to obtain the relative error $\epsilon_{\langle S\rangle}$. We have studied the dependence of the above magnitudes in terms of the transition (desorption) probability over (from) the surface boundary, the confining domain dimensions, and the finite transition probability at the escape window. Regarding the relative errors we observe that, roughly speaking, $1<\epsilon_{\langle t\rangle}<2$, which is in agreement with the noncompact exploration case defined in [22]. Alternatively we also present $S$ (and its relative error $\epsilon_{\langle S\rangle}$ ), a magnitude that has been proved to be reliable and may be considered in order to fully characterize the efficiency of the mixed transport.

The current approach can be generalized in several directions: non-Markovian desorption from surfaces, "dynamical" behavior of the escape window, etc. All of these aspects will be the subject of future work.

\section{ACKNOWLEDGMENTS}

F.R. acknowledges fruitful discussions with L. E. Aguirre, A. D. Dente, and M. P. Pedro. F.R., C.E.B. Jr., and CEB acknowledge support by CONICET and SeCyT (Universidad Nacional de Córdoba), Argentina. H.S.W. acknowledges financial support from MICINN, Spain, through Project No. FIS2010-18023.
[1] M. G. E. da Luz, A. Grosberg, E. P. Raposo, and G. M. Viswanathan, J. Phys. A: Math. Theor. 42, 430301 (2009).

[2] P. G. de Gennes, Adv. Colloid Interface Sci. 27, 189 (1987); J. F. Douglas, H. E. Johnson, and S. Granick, Science 262, 2010 (1993); B. L. Carvalho, P. Tong, J. S. Huang, T. A. Witten, and L. J. Fetters, Macromolecules 26, 4632 (1993).

[3] O. V. Bychuk and B. O’Shaughnessy, Phys. Rev. Lett. 74, 1795 (1995); J. H. Clint, Surfactant Aggregation (Chapman and Hall, New York, 1992).

[4] A. V. Chechkin, I. M. Zaid, M. A. Lomholt, I. M. Sokolov, and R. Metzler, Phys. Rev. E 79, 040105 (2009); G. Tkačik and W. Bialek, ibid. 79, 051901 (2009); M. A. Lomholt, B. van den Broek, S.-M. J. Kalisch, G. J. L. Wuite, and R. Metzler, Proc. Natl. Acad. Sci. USA 106, 8204 (2009).

[5] J. A. Revelli, C. E. Budde, D. Prato, and H. S. Wio, Eur. Phys. J. B 36, 245 (2003); J. A. Revelli, C. E. Budde, and H. S. Wio, J. Phys.: Condens. Matter 17, S4175 (2005); J. A. Revelli, C. E. Budde, D. Prato, and H. S. Wio, New J. Phys. 7, 16 (2005); J. A. Revelli, C. E. Budde, and H. S. Wio, J. Phys.: Condens. Matter 19, 065127 (2007)

[6] A. Honciuc, A. W. Harant, and D. K. Schwartz, Langmuir 24, 6562 (2008); R. Walder and D. K. Schwartz, ibid. 26, 13364 (2010); R. Walder, N. Nelson, and D. K. Schwartz, Phys. Rev. Lett. 107, 156102 (2011); Nat. Commun. 2, 515 (2011).

[7] S. Dupraz, D. Grassi, M. E. Bernis, L. Sosa, M. Bisbal, L. Gastaldi, I. Jausoro, A. Cáceres, K. H. Pfenninger, and S. Quiroga, J. Neurosci. 29, 13292 (2009).

[8] O. Bénichou, D. Grebenkov, P. Levitz, C. Loverdo, and R. Voituriez, J. Stat. Phys. 142, 657 (2011); Phys. Rev. Lett. 105, 150606 (2010).

[9] F. Rojo and C. E. Budde, Phys. Rev. E 84, 021117 (2011); F. Rojo, H. S. Wio, and C. E. Budde, ibid. 86, 031105 (2012).
[10] With "perfect or imperfect trapping" we refer to the particle's possibility of returning to the system, i.e., for the former there is none while for the second depends on the imperfection strength.

[11] T. Calandre, O. Bénichou, D. S. Grebenkov, and R. Voituriez, Phys. Rev. E 85, 051111 (2012).

[12] J.-F. Rupprecht, O. Bénichou, D. Grebenkov, and R. Voituriez, J. Stat. Phys. 147, 891 (2012); Phys. Rev. E 86, 041135 (2012).

[13] This terminology matches the one used in the NET area. See [9] and references therein.

[14] J. W. Haus and K. W. Kehr, Phys. Rep. 150, 263 (1987).

[15] M. A. Ré and C. E. Budde, Phys. Rev. E 61, 1110 (2000).

[16] W. Nadler and D. L. Stein, J. Chem. Phys. 104, 1918 (1996).

[17] We will denote an integral transform by its argument. Thus, for example, the Laplace transform on $t$ and the (finite) Fourier transform on a coordinate (e.g., $x)$ would read $P\left(k, m, l, u \mid n_{0}, m_{0}, l_{0}, 0\right)=$ $\sum_{n=0}^{N-1} e^{i k n} \int_{0}^{\infty} e^{-u t} P\left(n, m, l, t \mid n_{0}, m_{0}, l_{0}, 0\right) d t$.

[18] F. Rojo, P. A. Pury, and C. E. Budde, Physica A 389, 3399 (2010).

[19] Analytical results: $\langle t\rangle, \epsilon_{\langle t\rangle}, S(u)$; analytical numerical results: $S(u) \rightarrow S(t)$.

[20] We chose $\left(0,0, l_{0}\right)$ as the starting point just to fix some parameters. However, we are able to evaluate the magnitudes for any starting point.

[21] We follow a similar reasoning to that used in [9] and we remark that $\left.\Delta f_{1}\right|_{\mathrm{j}}$ does not depend on $\delta$ or $\alpha_{0}$.

[22] O. Bénichou, C. Chevalier, J. Klafter, B. Meyer, and R. Voituriez, Nat. Chem. 2, 472 (2010); B. Meyer, C. Chevalier, R. Voituriez, and O. Bénichou, Phys. Rev. E 83, 051116 (2011).

[23] We have chosen $t_{L}^{\prime}=\min \left(\langle t\rangle_{L}\right)$ where $\langle t\rangle_{L}$ are those from Fig. 2. Although this choice may seem arbitrary we have evaluated $S$ for different $t$ values, greater and lower than $\min \left(\langle t\rangle_{L}\right)$, and it preserves the behavior, except when is such large that $S^{*}=1$ $(S=N M)$. 